\title{
X-ray measurement of the elemental abundances at the outskirts of the Perseus cluster with Suzaku
}

\author{
S. Ueda ${ }^{1, \star}$, K. Hayashida ${ }^{1}$, H. Nakajima ${ }^{1}$, and H. Tsunemi ${ }^{1}$ \\ Department of Earth and Space Science, Graduate School of Science, Osaka University, Toyonaka, Osaka, 560-0043, \\ Japan
}

Received 24 August 2012, accepted 16 October 2012

Published online later

Key words galaxies: clusters: individual (Perseus cluster) - X-rays: galaxies: clusters

\begin{abstract}
We report on the abundance of metals $\left(\mathrm{Mg}\right.$ and Fe) in the intracluster medium (ICM) at the outskirts $\left(0.2 r_{200}-0.8 r_{200}\right)$ of the Perseus cluster. The X-ray spectra were obtained in the Suzaku/XIS mapping observations of this region. We employ single temperature models to fit all the X-ray spectra. The ICM temperature smoothly decreases toward the outer region from $6 \mathrm{keV}$ to $4 \mathrm{keV}$. The $\mathrm{Fe}$ abundance is uniformly distributed at the outskirts $(\sim 0.3$ solar $)$. The Mg abundance is $\sim 1$ solar at the outskirts. The solar ratios of $\mathrm{Mg} / \mathrm{Fe}$ of the outskirts region $(\mathrm{Mg} / \mathrm{Fe} \sim 4)$ are a factor of 4 larger than those of the central region. Various systematic effects, including the spatial fluctuations in the cosmic X-ray background, are taken into account and evaluated. These our results have not changed significantly.
\end{abstract}

(c) 2012 WILEY-VCH Verlag GmbH \& Co. KGaA, Weinheim

\section{Introduction}

Metals in the intracluster medium (ICM) was discovered by Mitchell et al. (1976) who detected He-like Fe lines in the Perseus cluster for the first time. The metal abundance in the ICM has been measured in a number of clusters with its spatial distribution. Matsushita (2011) reported that Fe abundance distribution is centrally peaked in clusters with a cD galaxy, indicating a significant amount of metals at cluster cores is supplied from those $\mathrm{cD}$ galaxies. Recent X-ray observations extended the area of the metallicity measurement to and beyond virial radii (e.g. Fujita et al. 2008, Simionescu et al. 2011, and Urban et al. 2011); metals are distributed at the virial radii of clusters with typical abundance (primarily determined from $\mathrm{Fe}$ ) of 0.3 solar. These results suggest that the ICM is not a pure pristine gas but polluted by metals from galaxies even in the outskirts of clusters.

Significant fraction of metals in the ICM are generated by supernovae. Type Ia supernovae (SNe Ia) produce a large amount of Fe, while Type II supernovae (SNe II) synthesize lighter elements than $\mathrm{Fe}$ such as $\mathrm{O}, \mathrm{Ne}$, and $\mathrm{Mg}$. The abundance of each light element $(\mathrm{O}, \mathrm{Ne}$, and $\mathrm{Mg})$ in the ICM was measured in the core of some bright clusters (e.g. Sato et al. 2008, Tamura et al. 2009, and Sakuma et al. 2011). On the other hand, measurements of the metal abundances at the cluster outskirts have been limited to Fe. Therefore contribution of each type of $\mathrm{SNe}$ is difficult to be evaluated for them.

Suzaku, the 5th Japanese X-ray astronomy satellite, carries the X-ray Imaging Spectrometer (XIS: Koyama et al. 2007) consisting of four X-ray CCD cameras. Advantages

\footnotetext{
* Corresponding author: e-mail: shutaro@ess.sci.osaka-u.ac.jp
}

of the XIS include its large effective area, low non X-ray background (NXB), and good reproducibility of the NXB. Therefore, the XIS is a powerful tool for observing faint and diffuse objects such as the outskirts of clusters as already demonstrated (e.g. Hoshino et al. 2010, Simionescu et al. 2011).

The Perseus cluster, as the X-ray brightest and one of the nearest clusters, is the best target to measure the spatial distribution of various elements in the ICM. Dupke \& Arnaud (2001) measured the $\mathrm{Fe}$ abundance at the outer region $\left(25^{\prime}-45^{\prime}\right)$ using ASCA. Tamura et al. (2009) discovered rare elements ( $\mathrm{Cr}$ and $\mathrm{Mn}$ ) in the core using Suzaku/XIS. Simionescu et al. (2011) reported the large scale structure of the ICM beyond virial radius with Suzaku/XIS. They reported that the metallicity of the ICM at the outskirts is $\sim 0.3$ solar throughout the virial radius, although it is measured commonly for all the elements, predominantly determined by the $\mathrm{Fe}$ abundance. Mapping observations of the Perseus cluster have been extensively performed, and the area covered is several times larger than those used in Simionescu et al. (2011). We focus on the $\mathrm{Mg}$ and $\mathrm{Fe}$ abundances of the ICM at the outskirts of the Perseus cluster $\left(0.2 r_{200}-0.8 r_{200}\right)$.

In this paper, we adopt the abundance table of Anders $\&$ Grevesse (1989) and the Hubble constant of $H_{0}=70 \mathrm{~km}$ $\mathrm{s}^{-1} \mathrm{Mpc}^{-1} .1$ arcmin corresponds to $22 \mathrm{kpc}$ at the redshift of this cluster, i.e., 0.0183 , and the virial radius $\left(r_{200}\right)$ is $1.79 \mathrm{Mpc}$ (Simionescu et al. 2011). Unless otherwise specified, all errors represent at 90\% confidence level (90\% CL). 


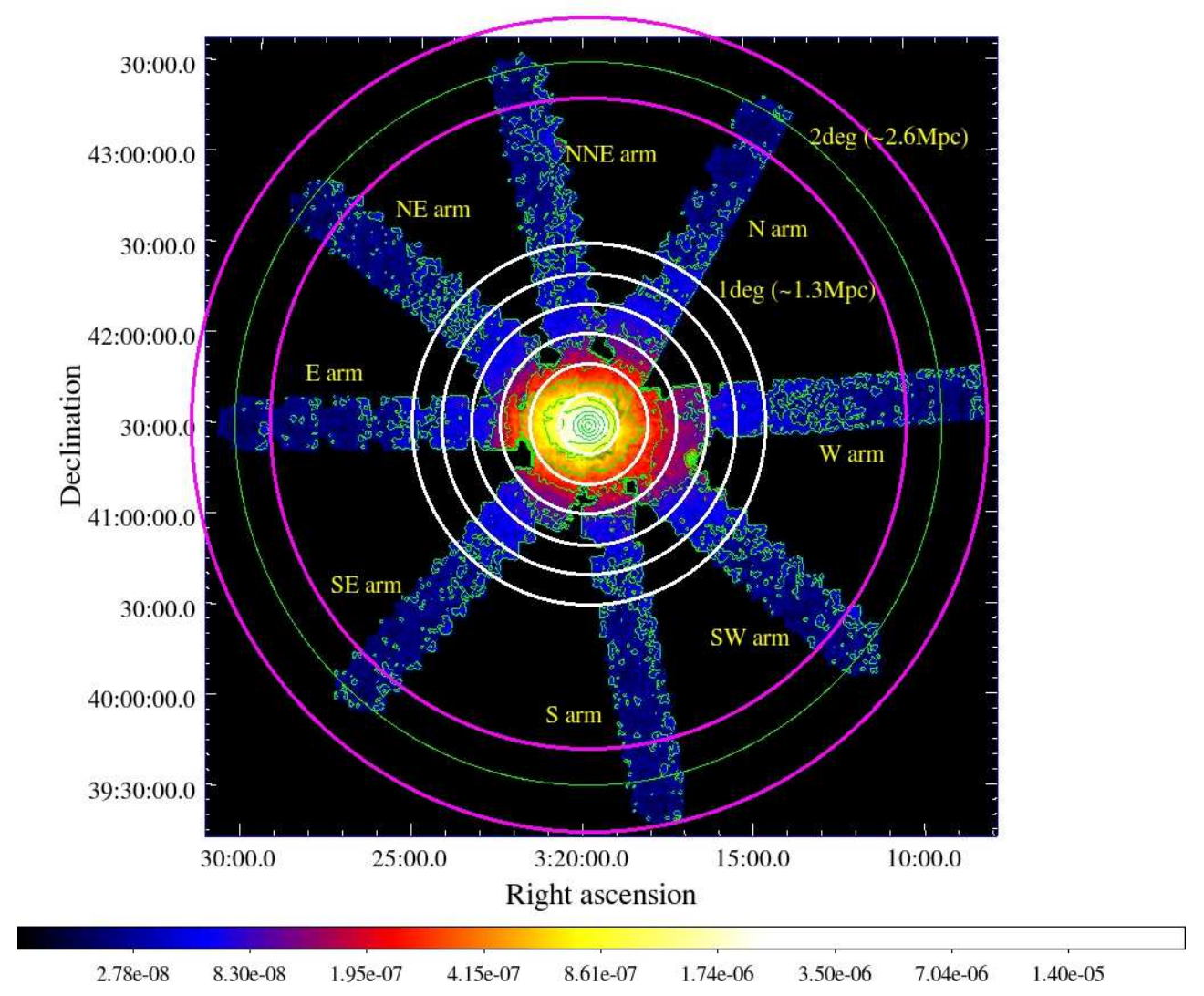

Fig. 1 X-ray image of the Perseus cluster. Vignetting and exposure corrections were applied. The NXB was not subtracted, though its contribution is smaller than $30 \%$ of the total flux. The unit of color bar is counts $\mathrm{s}^{-1}$ pixel $^{-1}$. X-ray spectra were extracted from 5 annular regions bordered with white circles (their radius ranges from $10^{\prime}$ to $60^{\prime} \sim 1.3 \mathrm{Mpc}$ ). The CXB and the Galactic emissions were estimated from the X-ray spectra extracted from the regions in the magenta annulus, whose distance is about $2.6 \mathrm{Mpc}$ from the center.

\section{Observations and data reduction}

Suzaku mapping observations of the Perseus cluster were performed from July 2009 to September 2011 (PI: S. W. Allen). These observations form 8 arms, each of which consists of $7-12$ pointings. Fig. 1 shows the X-ray image of the Perseus cluster. Only the observations of the $\mathrm{N}$ and $\mathrm{E}$ arms in Fig. 1 were employed in Simionescu et al. (2011). Although each arm extends to $2^{\circ}\left(2.6 \mathrm{Mpc}\right.$ or $\left.1.6 r_{200}\right)$ from the center, we extracted the X-ray spectra from the region of $10^{\prime} \leq r \leq 60^{\prime}$, dividing it into 5 annuli in steps of $10^{\prime}$, as shown in Fig. 1 with white circles. The outermost radius of $60^{\prime}$ corresponds to $0.8 r_{200}$. We excluded a circular region with radius of $5^{\prime}$ around the the bright X-ray source IC 310 .

\section{Spectral analyses}

We employed the emission model of thin thermal plasma with a single temperature (vAPEC, Smith et al. 2001) to reproduce the X-ray spectra of the ICM. The NXB was estimated by using the database of night earth observations with xisnxbgen (Tawa et al. 2008) and subtracted. The cosmic $\mathrm{X}$-ray background (CXB) and the Galactic emissions were included as spectral models.

\subsection{Components of the CXB and the Galactic emissions}

The CXB and the Galactic emissions (MWH: Milky Way halo and LHB: local hot bubble) were reproduced with a cut-off power-law (PL) and a two-component APEC model, respectively. The schematic model was described as wabs $\times$ $\left(\right.$ vAPEC + cut-off PL $\left.+\mathrm{APEC}_{\mathrm{MWH}}\right)+\mathrm{APEC}_{\mathrm{LHB}}$, where wabs model represented the Galactic absorption using Wisconsin cross-sections (Morrison \& McCammon 1983). We fixed $N_{\mathrm{H}}$ at $0.132 \times 10^{22} \mathrm{~cm}^{-2}$, which was determined in the X-ray observations of the central region of this cluster (Nishino et al. 2010). This value is consistent with that from 21-cm observations (Kalberla et al. 2005).

We determined the CXB and the Galactic emissions by fitting the X-ray spectra of the outermost regions beyond $2.3 \mathrm{Mpc}$ (as shown with magenta annulus in Fig. 1). The cutoff energy of the cut-off PL for the CXB was fixed to $40 \mathrm{keV}$, according to Boldt (1987). The photon index and the flux of cut-off PL are $1.43 \pm 0.03$ and $2.37 \pm 0.08 \times 10^{-11} \mathrm{ergs} \mathrm{cm}^{-2}$ $\mathrm{s}^{-1} \mathrm{deg}^{-2}(2-10 \mathrm{keV})$, respectively. These are consistent with those reported by Moretti et al. (2009). For the Galactic emissions, the abundance was set to 1 solar for both mod- 


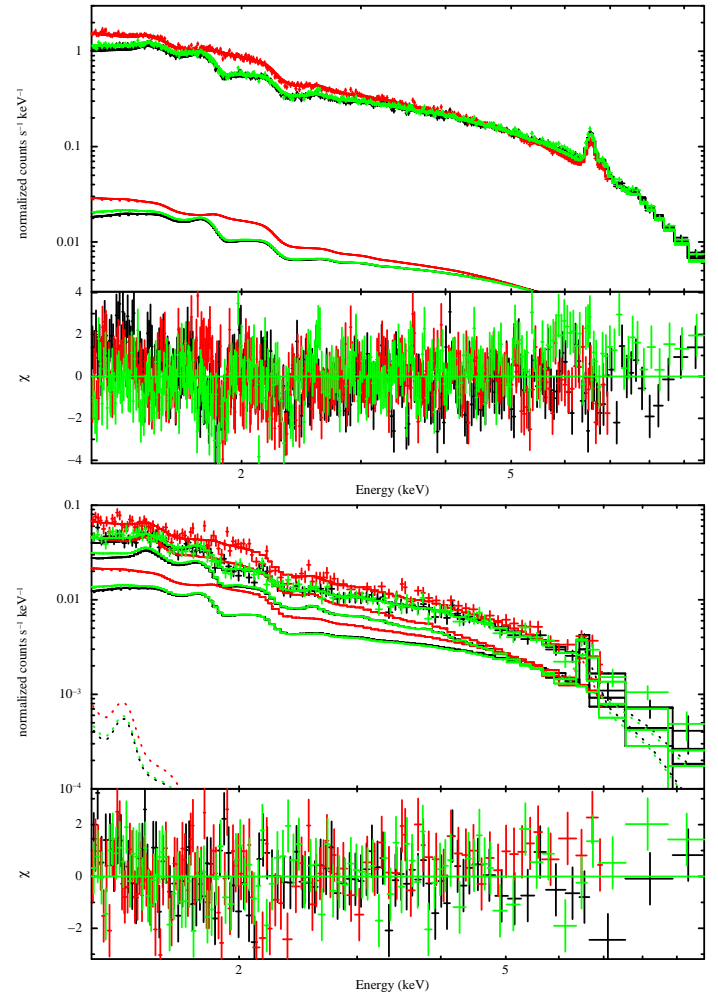

Fig. 2 Top: X-ray spectra of the innermost region of our analyses $\left(10^{\prime}-20^{\prime}\right)$. Single temperature model (vAPEC) is used for the ICM components in the fit. The CXB and the Galactic emissions were modeled as mentioned in 3.1. The spectra are plotted by black, red, and green across for those of XIS0, XIS1, and XIS3, respectively. Bottom: Same as the top panel but for the outermost region $\left(50^{\prime}-60^{\prime}\right)$.

els. The gas temperatures are $0.23 \pm 0.02 \mathrm{keV}(\mathrm{MWH})$ and $0.07 \pm 0.02 \mathrm{keV}$ (LHB), respectively.

\subsection{ICM component}

For the ICM (vAPEC) component, the abundances of He, $\mathrm{C}, \mathrm{N}, \mathrm{O}, \mathrm{Ne}$, and $\mathrm{Al}$ were fixed to 1 solar, and $\mathrm{Ni}$ was linked to Fe. We fitted the X-ray spectra in $1.2-10.0 \mathrm{keV}$ at XIS0, 3 and $1.2-7.0 \mathrm{keV}$ at XIS1 to avoid uncertainties of $\mathrm{N}_{\mathrm{H}}$ and the contamination on OBF (Koyama et al. 2007). We focus on $\mathrm{Mg}$ and $\mathrm{Fe}$ abundances in this paper. The top panel of Fig. 2 shows the X-ray spectra at the innermost region $\left(10^{\prime}-20^{\prime}\right)$. The bottom panel of Fig. 2 shows those of the outermost region $\left(50^{\prime}-60^{\prime}\right)$. The $\chi^{2} /$ d.o.f. of the innermost region is $1243 / 851$ and that of the outermost region is $460 / 389$.

\section{Results}

The radial profile of the ICM temperature is shown in the left panel of Fig. 3. The ICM temperatures of the central regions $\left(\leq 10^{\prime}\right)$ are taken from Tamura et al. (2009). The ICM temperature smoothly decreases from $6 \mathrm{keV}$ to $4 \mathrm{keV}$ toward the outside. This result is consistent with that obtained by Simionescu et al. (2011).

We measured the $\mathrm{Mg}$ and $\mathrm{Fe}$ abundances in $0.2 r_{200}-$ $0.8 r_{200}$ region. The radial profiles of the $\mathrm{Mg}$ and $\mathrm{Fe}$ abundances are shown in the middle panel and the right panel of Fig. 3, respectively. The Fe abundance is uniformly distributed at the outskirts ( $\sim 0.3$ solar). The Mg abundance is $\sim 1$ solar in the same region. We take the solar ratio of $\mathrm{Mg}$ abundance to that of the $\mathrm{Fe}(\mathrm{Mg} / \mathrm{Fe}$ ratio) in order to minimize the systematic errors to uncertainties in the plasma model for Fe-L complex. The result is shown in Fig. 4. The mean values of $\mathrm{Mg} / \mathrm{Fe}$ ratios of the central regions is $0.95 \pm 0.11$, while that of the outskirts is $3.7 \pm 0.8$. We carried out the $t$-test in order to verify the statistical difference between these two value. These two values are statistically different, i.e., the hypothesis that these two values are the same is rejected at a significance level of 0.001 .

We evaluated the systematic errors owing to the spatial fluctuation of the CXB flux in our results. Tawa et al. (2008) estimated that the fluctuation in the CXB flux is $14 \%$ $(1 \sigma)$ for the whole area of the XIS. Assuming the fluctuation has been inversely proportional to the root mean square of the size of the sky area, the magnitude of the fluctuation for each annular region was calculated. We added and subtracted the $90 \% \mathrm{CL}$ of the fluctuation to the CXB nominal flux model (as mentioned in 3.1) when we fitted the $\mathrm{X}$-ray spectra of in each annular region. As shown in Fig. 3 (light blue diamonds for $+90 \% \mathrm{CL}$, those of magenta for $-90 \% \mathrm{CL}$ ), the systematic errors due to the CXB fluctuations are much smaller than the statistical errors in the radial profiles of the ICM temperature, the $\mathrm{Mg}$, and $\mathrm{Fe}$ abundance.

We also examined the systematic errors due to fixed the abundances of $\mathrm{C}, \mathrm{N}, \mathrm{O}, \mathrm{Ne}$, and $\mathrm{Al}$ on the abundances of $\mathrm{Mg}$ and $\mathrm{Fe}$. We fixed the abundances of these elements at 1 solar. We tested the spectral analysis of $10^{\prime}-20^{\prime}$ region by changing the abundances of these elements to 0.5 solar and 2 solar. This changes the best fit values of the Mg and Fe abundance by at most $13 \%$. We also checked the possibility that the $\mathrm{Al}$ abundance affects the $\mathrm{Mg}$ abundance. Even if we take the extreme case, i.e., 0.1 solar and 10 solar for the $\mathrm{Al}$ abundance, the $\mathrm{Mg}$ abundance differs by $15 \%$. These systematic effect cannot explain the factor of 4 difference in the $\mathrm{Mg} / \mathrm{Fe}$ ratios we observed. Note that although we fixed the He abundance at 1 solar, as has been commonly assumed, changing this value scales all abundances of elements heavier than C (e.g. Ettori \& Fabian 2006).

Another concern is the difference in the emission model we assumed, i.e., single temperature or two temperatures. Nevertheless, it is found that the $\mathrm{Mg}$ abundance in the central $\left(4^{\prime}-10^{\prime}\right.$ region) is altered by at most $+21 \%$ if we fit the spectra with a single temperature model, instead of the two-temperature model.

The Fe abundance in the ICM is centrally peaked, while that of the outskirts is almost constant. This result suggests that significant amount of $\mathrm{Fe}$ is supplied from the cD galaxy where the contribution of SNe Ia is relatively large. Larger 

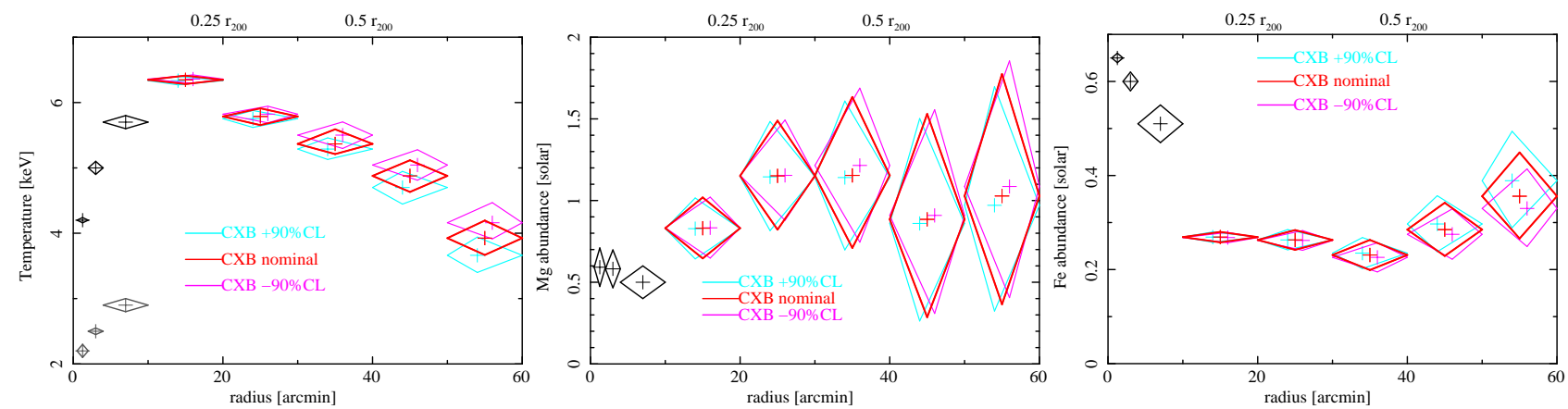

Fig. 3 Left: Radial profile of the ICM temperature including systematic errors ( $\pm 90 \% \mathrm{CL}$ ) owing to the spatial fluctuation of the CXB. Middle: Same as the left panel but for the Mg abundance. Right: Same as the central panel but for the Fe abundance. The black diamonds in the inner regions $\left(\leq 10^{\prime}\right)$ are taken from Tamura et al. (2009). The red diamonds show the temperature, the $\mathrm{Mg}$ and Fe abundances of the ICM in each annular region with the nominal flux of the CXB. The light blue and magenta diamonds show the results when we changed the $+90 \%$ CL or $-90 \%$ CL of the CXB flux, respectively.

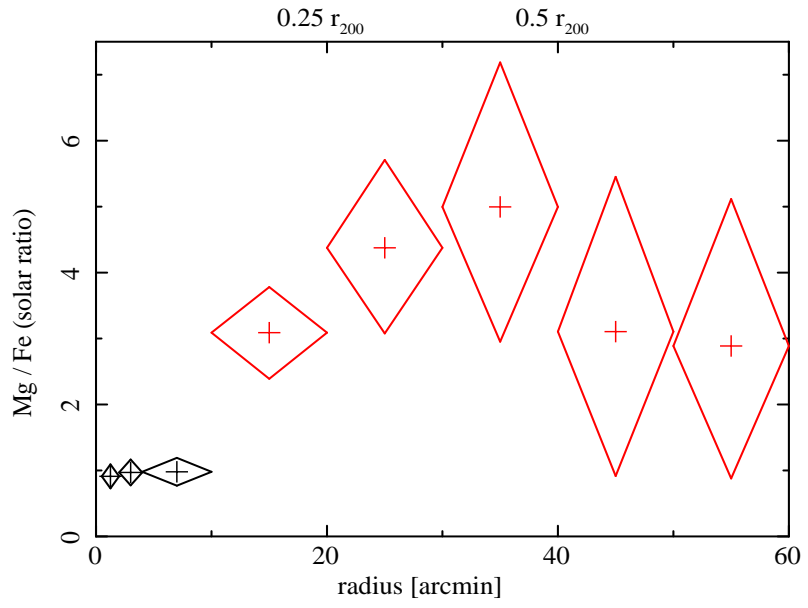

Fig. 4 Radial profile of the solar ratio of the $\mathrm{Mg}$ abundance to the Fe abundance.

$\mathrm{Mg} / \mathrm{Fe}$ ratio at the outskirts than that in the central region is another support on this point of view.

\section{Summary}

We summarize our results on the ICM at the outskirts of the Perseus cluster as follows:

1. We measured the metal abundances ( $\mathrm{Mg}$ and $\mathrm{Fe})$ separately at $0.2 r_{200}-0.8 r_{200}$.

2. The Fe abundance is constant of $\sim 0.3$ solar at the outskirts.

3. The $\mathrm{Mg}$ abundance is $\sim 1$ solar in the outskirts region.

4. The mean value of $\mathrm{Mg} / \mathrm{Fe}$ ratio is $0.95 \pm 0.11$ in the central region, while that in the outskirts is $3.7 \pm 0.8$. This difference is significant even we consider the possible systematic effects. Centrally peaked Fe abundance is likely due to large contribution of SNe Ia products from the $\mathrm{cD}$ galaxy. The difference in the $\mathrm{Mg} / \mathrm{Fe}$ ratio we observe is consistent with this point of view.
Acknowledgements. We are grateful to the anonymous referee for helpful suggestions and comments. We thank all members of the Suzaku operation team and the XIS calibration team. We also thank A. Simionescu, N. Werner, Y. Takei, and T. Tamura for their useful comments. SU is supported by Japan Society for the Promotion of Science (JSPS) Research Fellowship for Young Scientist (A2411900). This work was also supported by JSPS KAKENHI Grand Number 23340071 (KH), 24684010 (HN), and 23000004 (HT).

\section{References}

Anders, E. \& Grevesse, N.: 1989, Geochim. Cosmochim. Acta, 53, 197

Boldt, E.: 1987, Phys. Rep., 146, 215

Dupke, R. A., \& Arnaud, K. A.: 2001, ApJ , 548, 141

Ettori, S., \& Fabian, A. C.: 2006, MNRAS , 369, L42

Fujita, Y., et al.: 2008, PASJ , 60, 343

Hoshino, A., et al.: 2010, PASJ , 62, 371

Kalberla, P. M. W., et al.: 2005, A\&A , 440, 775

Koyama, K., et al: 2007, PASJ , 59, 23

Matsushita, K.: 2011, A\&A , 527, A134

Moretti, A., et al.: 2009, A\&A , 493, 501

Morrison, R., \& McCammon, D.: 1983, ApJ , 270, 119

Mitchell, R. J., et al.: 1976, MNRAS , 175, 29P

Nishino, S., et al.: 2010, PASJ , 62, 9

Sakuma, E., et al.: 2011, PASJ , 63, 979

Sato, K., et al.: 2008, PASJ , 60, S333

Simionescu, A., et al.: 2011, Science, 331, 1576

Smith, R. K., et al.: 2001, ApJ , 556, L91

Tamura, T., et al.: 2009, ApJ , 705, L62

Tawa, N., et al.: 2008, PASJ, 60, S11

Urban, O., et al.: 2011, MNRAS , 414, 2101 